\title{
Meta analysis: An insight
}

\author{
S C Mohapatra ${ }^{1 *}$, Sunil Kumar Chamola ${ }^{2}$
}

${ }^{1}$ Dean Academic Affairs and Professor \& Head Community Medicine, ${ }^{2}$ Statistitian cum Lecturer; Dept. of Community Medicine, Faculty of Medicine \& Health Sciences, SGT University, Gurgaon, Haryana, India

*Corresponding Author: S C Mohapatra

Email: vishwamegh@gmail.com

\section{Introduction}

Meta analysis is used when comparing of multiple studies that looked as similar are consolidated using statistical method. It is a quantitative approach for systematically combing results of previous research to arrive at a convergent conclusion.

The historically meta-analysis dates back to 17 th century studies of astronomy. ${ }^{1}$ Karl Pearson in 1904 mooted the idea of Meta analysis collating data of several studies of typhoid inoculation and used the information to aggregate from the outcomes of multiple clinical studies. ${ }^{2}$ The first meta-analysis was on Extrasensory Perception After Sixty Years, by J. G. Pratt, J. B. Rhine of Duke in $1940 .^{3}$ In the 1970s, more sophisticated analytical techniques were introduced Meta analysis by Gene V. Glass, Frank L. Schmidt and John E. Hunter.

The Greek root 'meta' means 'with', 'along', 'after', or 'later' and meta-analysis is done after original analysis. Glass defined meta-analysis as the statistical analysis of a large collection of analysis resulting from individual studies, in order to integrate the findings. ${ }^{4}$ Meta-analysis has a quantitative, formal, epidemiological study design. A meta-analysis is a statistical analysis that combines the results of multiple scientific studies, undertaken by same or different researchers at more than one places as well as being accessed from one or more journals.

\section{Advantages and disadvantages}

Conceptually, a meta-analysis combines the results from multiple studies in order to increase power of the data over individual studies. Naturally therefore there is improvement in estimates of the size of the effect to resolve uncertainty at the stage of disagreement. It produces a weighted average of the included study results. While by Meta analysis the results can be generalized to a larger population, the precision and accuracy of estimates can be improved and will increase the statistical power to detect an effect. Inconsistency of results across studies can also be quantified and analyzed by combining effect of Meta analysis. Hypothesis testing, summary estimates, moderation to explain variation between studies and publication bias can be investigated through Meta analysis. Greater statistical power, confirmatory data analysis, greater ability to extrapolate to general population affected and creation of an evidence- based resource material can be undertaken by Meta Analysis.

However has following in-built problems which are sometimes considered as the Disadvantages. It is difficult and time consuming to identify appropriate studies and create a meta modification of them. Further not all studies provide adequate data for inclusion and analysis and heterogeneity of study populations may create difficulty during data handling apart from the fact it needs advanced statistical techniques which many statisticians are not comfortable with.

A meta-analysis of several small studies does not predict the results of a single large study. ${ }^{5}$ Some have argued that a weakness of the method is that sources of bias are not controlled by the method: even a good meta-analysis may not correct for poor design or bias both, in the original studies. ${ }^{6}$ This would mean that only methodologically sound studies should be included in a meta-analysis, a practice called 'best evidence synthesis'. ${ }^{7}$ Other meta-analysts would include weaker studies, and add a study-level predictor variable that reflects the methodological quality of the studies to examine the effect of study quality on the effect size. ${ }^{8}$ However, others have argued that a better approach is to preserve information about the variance in the study sample, casting as wide a net as possible, and that methodological selection criteria introduce unwanted subjectivity, defeating the purpose of the approach. ${ }^{9}$

\section{Steps in carrying out meta- analysis: $:^{5}$}

According to Glass, the meta-analyst (a) uses objective methods to find studies for a review; (b) describes the features of the studies in quantitative or quasi-quantitative terms; (c) expresses treatment effects of all studies on a common scale of effect size; and (d) uses statistical techniques to relate study features to study outcomes ${ }^{\text {[loc.cit] }}$ In general, two types of evidence can be distinguished when performing a meta-analysis: individual participant data (IPD), and aggregate data (AD). The aggregate data can be direct or indirect. AD is more commonly available (e.g. from the literature) and typically represents summary estimates such as odds ratios or relative risks. IPD evidence represents raw data as collected by the study centers

WHO book on Basic Epidemiology suggests the following steps:

1. Formulating the problem and study design

2. Identifying relevant studies

3. excluding poorly conducted studies or those with major methodological flaws and

4. Measuring, combing and interpreting the results

An example of, Meta-analysis of the relative risk of cleft palate in the offspring of mothers who smoked during pregnancy were compared with the offspring of the women who did not smoke, has been depicted in the figure given below. At odds ratio of 1 the $95 \%$ confidence of all studies 


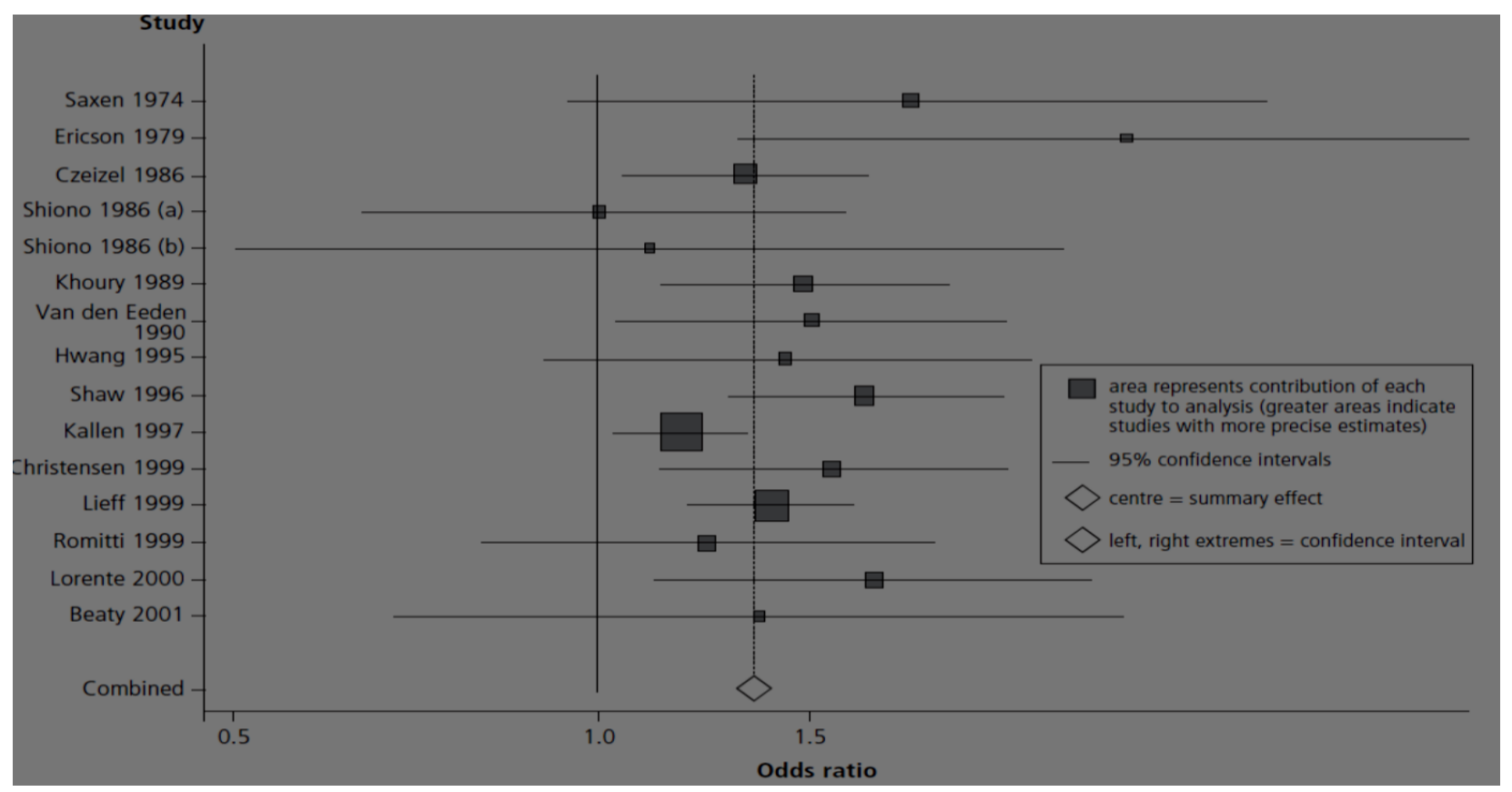

have been plotted. The studies have been indicated on the $y$ axis while the $\mathrm{X}$ axis shows the odds of the studies. The figure shows the serial odds as depicted by horizontal straight line. The Black squires show the precise odds area. Bigger the size of the squire, higher the precision. The white diamond shows the mean of all studies redesigned as per their odds and mean value. The lines on each side of the mean show the confidential interval. It clearly shows a higher Odds value for cleft palate vis-à-vis smoking. Thus cleft palate in the offspring of mothers who smoked during pregnancy has been one of the approved risk factors (vide infra).

To sum up, meta-analyses offer a systematic and quantitative approach in synthesizing evidence to answer important therapeutic questions. This method is statistically correct and epidemiologically predictable for multiple studies to reach at a conclusion. It should not simply be a summary of a literature, but should provide a theoretical interpretation and statistical integration. In general, the more a meta-analysis provides beyond its statistical calculations the more valuable its scientific contribution appears. Metaanalysis offers a rational and helpful way of dealing with a number of practical difficulties that beset anyone trying to make sense of effectiveness research.

\section{References}

1. Plackett RL. Studies in the history of probability and statistics: vii. The principle of the arithmetic mean. Biometrika. 1958;45(1-

2):133. Doi:10.1093/biomet/45.1-2.130. Retrieved 29 May 2016

2. Pearson K. Report on certain enteric fever inoculation statistics". BMJ 1904;2(2288):1243-

6. doi:10.1136/bmj.2.2288.1243. PMC 2355479

3. Pratt JG, Rhine JB, Smith BM, Stuart CE, Greenwood JA. Extra-Sensory Perception after Sixty Years: A Critical
Appraisal of the Research in Extra-Sensory Perception. New York: Henry Holt, 1940.

4. Glass GV. Primary, secondary and meta-analysis of research. Educational Researcher. 1976;5:3-8.

5. Bonita R, Beaglehole R, Kjellstrom, Basic Epidemiology ${ }^{\text {nd }}$ Edition WHO;2006.

6. LeLorier J, Grégoire G, Benhaddad A, Lapierre J, Derderian F. Discrepancies between Meta-Analyses and Subsequent Large Randomized, Controlled Trials". New Engl J Med 1997;337(8):53654.

7. Slavin RE. Best-Evidence Synthesis: An Alternative to MetaAnalytic and Traditional Reviews". Educational Researcher. 1986;15(9):5.

8. Hunter, Schmidt, \& Jackson, John E.). Meta-analysis: Cumulating research findings across studies. Beverly Hills, California: Sage. 1982

9. Glass, McGaw, \& Smith. Meta-analysis in social research. Beverly Hills, CA: Sage, 1981.

How to cite this article: Mohapatra SC, Chamola SK. Meta analysis: An insight. J Community Health Manag 2019;6(3):66-7. 